\title{
Aspectos clínicos e histopatológicos do Lúpus Eritematoso Discoide canino: relato de caso
}

\author{
[Clinical and histopathological aspects of canine Discoid Lupus Erythematosus: case report]
}

\section{"Relato de Caso/Case Report"}

\author{
Stphanie Larissa Ramos de Santana Leal*, Joelma Gomes da Silva, Moisés Dantas Tertulino, \\ Giovana Meireles Fixina Barreto, Jéssyka Araújo Noronha, Lívia Maria Nascimento Rodrigues, \\ Natália Cristina de Medeiros
}

Universidade Federal Rural do Semi-Árido Mossoró-RN, Brasil.

*Autora para correspondência/Corresponding author. E-mail: stphaniemedvet@gmail.com

\section{Resumo}

O Lúpus Eritematoso Discoide (LED) é uma dermatopatia autoimune. Sua etiologia ainda não está completamente elucidada, porém é pressuposto que se trata de uma doença multifatorial. Objetivou-se, neste trabalho, relatar um caso de LED em um cão sem raça definida, idoso, atendido no Hospital Veterinário Jerônimo Dix-Huit Rosado Maia, Mossoró - RN. O paciente apresentava lesão em ponte nasal com presença de ulcerações e crostas de evolução gradativa em um período de dois meses. Foram realizados exame histopatológico e diagnóstico diferencial para leishmaniose visceral canina por meio de ELISA e RIFI, confirmando LED. O protocolo terapêutico incluiu medicação tópica à base de hidrocortisona (1\%), vitamina E $(0,5 \%)$ e fator de proteção 45 a cada 12 horas por 20 dias; a terapia sistêmica se deu através da administração de prednisolona $(1 \mathrm{mg} / \mathrm{kg}$ a cada 24 horas, durante 20 dias; $0,75 \mathrm{mg} / \mathrm{kg}$, a cada 24 horas, durante 20 dias; $0,5 \mathrm{mg} / \mathrm{kg}$, a cada 24 horas, durante 20 dias, seguido de $0,25 \mathrm{mg} / \mathrm{kg}$, durante 20 dias; até retirada completa do medicamento), protetor hepático ( 2 comprimidos, a cada 24 horas durante 30 dias), além de precauções quanto à exposição solar. Após quatro meses, o paciente apresentou remissão completa, sem presença de recidiva mesmo após a suspensão do tratamento, mantendo-se sem recidivas após 8 meses do término do protocolo terapêutico. Recomendou-se usar protetor solar fator 45 por toda a vida do animal. Deste modo, concluiu-se que o protocolo estabelecido é eficaz para o tratamento de LED, salientando a importância da realização de exames complementares para obtenção do diagnóstico definitivo.

Palavras-chave: doença autoimune; biopsia; cão; corticoterapia; dermatopatia.

\begin{abstract}
Discoid Lupus Erythematosus (DLE) is an autoimmune dermatopathy; its etiology is not completely elucidated yet, however it is known to be a multifactorial disease. This work aims to report a case of DLE in a mixed-breed senior dog, admitted to Jerônimo Dix-Huit Rosado Maia Veterinary Hospital, in the city of Mossoró-RN. The patient presented with an erosive and crusting lesion on the bridge of the nose that have been showing gradual growth in a two-month period. Histopathological analysis and differential diagnosis for canine visceral leishmaniasis were performed throught ELISA and RIFI tests, however the DLE was confirmed. The treatment protocol included topical therapy hydrocortisone, vitamin E $(0.5 \%)$ and a sun protection factor 45 product, every twelve hours per 20 days; systemic therapy was made with prednisolone $(1 \mathrm{mg} / \mathrm{kg}$ every 24 hours per 20 days: $0.75 \mathrm{mg} / \mathrm{kg}$ every 24 hours per 20 days; following $0.25 \mathrm{mg} / \mathrm{kg}$ per 20 days, until complete withdrawal), hepatoprotector ( 2 pills, every 24 hours, during 30 days), as well as preventing sun exposure. After four months the patient presented complete disease remission, without relapsing, even after the treatment interruption, remaining without recurrences 8 months after the end of the therapeutic protocol. Use of the topical sun protection factor 45 product was recommended continuously. The report concluded that the protocol established is efficient to DLE treatment, demonstrating the importance of complementary exams in order to obtain a definitive diagnosis.
\end{abstract}

Keywords: autoimmune disease; biopsy; dog; corticotherapy; dermatopathy. 


\section{Introdução}

A pele, enquanto tecido linfoide terciário, pode apresentar falhas que contribuem para o aparecimento de enfermidades. Isto pode ser visto quando ocorre uma hiperatividade, por exemplo, e os fatores imunológicos favorecem o surgimento de patologias autoimunes ou imunomediadas, que geram respostas do tipo celular e humoral. No caso da produção de anticorpos, ocorre uma identificação de proteínas cutâneas como seus antígenos (Gomes, 2015).

Dentre as doenças autoimunes, o complexo lúpus eritematoso é uma das manifestações patológicas que mais acomete cães e, de maneira incomum, os gatos (Medleau e Hnilica, 2003; Palumbo et al., 2010). Esse complexo pode se manifestar das seguintes formas: lúpus eritematoso sistêmico (LES), lúpus eritematoso cutâneo esfoliativo (LECE), lúpus eritematoso cutâneo vesicular (LECV), lúpus eritematoso discoide (LED) e oniquite lupoide canina (Gomes, 2015; Banovic et al., 2017).

O Lúpus Eritematoso Discoide (LED), também denominado de Lúpus Eritematoso Cutâneo Crônico (LECC), pode apresentar ainda duas variantes: lúpus eritematoso discoide localizado facial (LEDL), que corresponde a uma forma localizada; e lúpus eritematoso discoide generalizado (LEDG), que pode atingir diferentes regiões do corpo. Na variação da LED, a sintomatologia é restritamente cutânea, podendo ser localizada ou disseminada, por isso é considerada benigna e seu prognóstico é favorável (Banovic, 2019). Com relação a sua etiologia, ainda é bastante controversa, tendo uma linha de pesquisa para genética, outra ambiental, com incidência dos raios UV, além do fator raça, em que diversos autores apontam mais casos em cães das raças Collie, Pastor Alemão, Shetland Sheepdogs, Huskie Siberiano, Brittany Spaniel e Pointer Alemão de pelo curto (Palumbo et al., 2010; Miller et al., 2013).

Todo esse quadro se torna de interesse clínico, ao passo que as dermatopatias autoimunes são raras em cães e gatos, e, dentro dessa realidade, o lúpus eritematoso discoide, que é a segunda mais frequente (Ferreira Filho et al., 2014), ainda apresenta muitos aspectos a serem esclarecidos; sendo, portanto, seu entendimento de grande contribuição para pesquisa diagnóstica e parâmetros clínicos. Diante disto, objetivou-se relatar um caso de lúpus eritematoso discoide em um cão.

\section{Descrição do Caso}

Um cão, macho, sem raça definida, resgatado há seis anos já adulto, tendo uma idade estimada em 12 anos e massa corpórea de 21,10 $\mathrm{kg}$ chegou para atendimento clínico no Hospital Veterinário Jerônimo Dix-Huit Rosado Maia (HOVET), Mossoró - RN. Na anamnese, a tutora relatou que o paciente, há dois meses, apresentou uma lesão na ponte nasal, com aumento gradativo, sem presença de prurido.

No exame físico, os linfonodos submandibulares, cervical superficial e poplíteos estavam aumentados. O paciente apresentava área de ulceração com crostas na região de ponte nasal, e presença de crostas nas pálpebras superiores (Figura 1). Foi percebido que o paciente apresentava estado nutricional de sobrepeso (grau 7 de índice de condição corporal para cães - ICC, baseado em Laflamme, 1997). Além do relatado, o paciente não apresentava outras alterações locais ou sistêmicas dignas de nota. Sendo assim, foram solicitados hemograma, exame bioquímico sérico hepático e renal e proteinograma, citologia de medula óssea e dos linfonodos cervical superficial e poplíteo para pesquisa de Leishmania sp., ensaio de imunoabsorção enzimática (ELISA) e imunofluorescência indireta (RIFI) para Leishmaniose Visceral Canina (LVC).

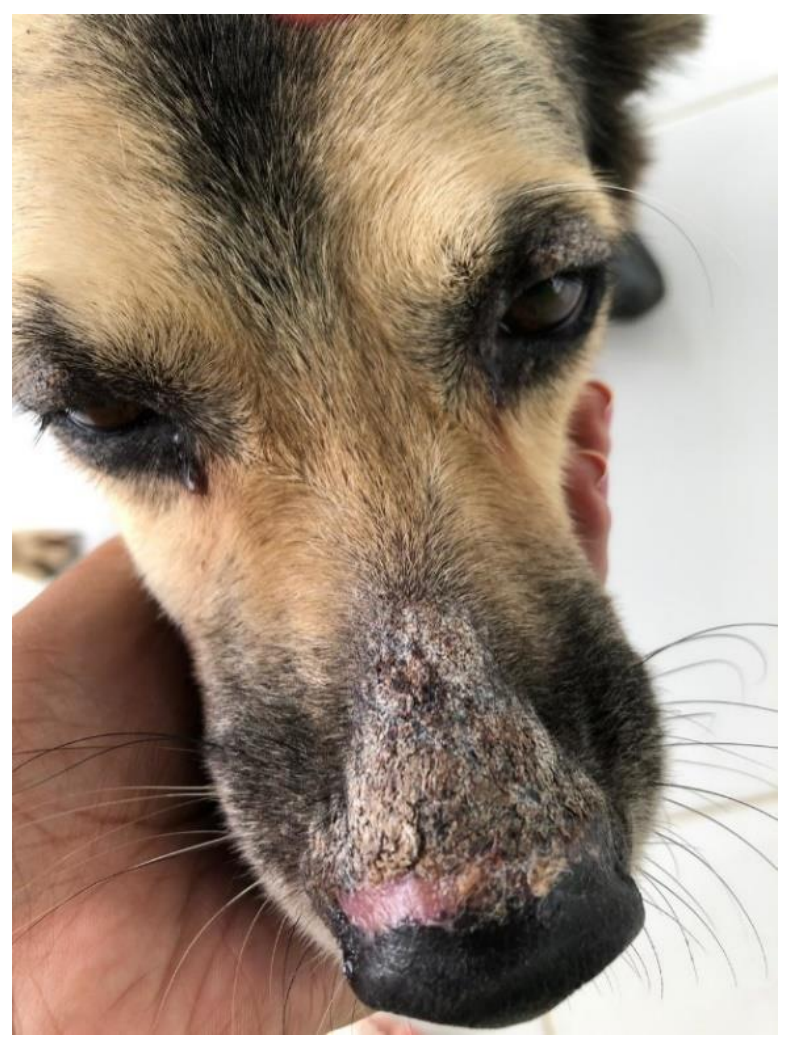

Figura 1. Cão, idoso, sem raça definida, apresentando área de ulceração e crostas na região de ponte nasal, e área de crostas na região de pálpebra superior. 
No hemograma e provas bioquímicas renais, o paciente não apresentou valores fora dos padrões de referência. Avaliações hepática e do proteinograma foram marcadas por alteração nos valores de Fosfatase Alcalina e proteínas totais, globulina e relação albumina/globulina (Tabela 1). $\mathrm{Na}$ citologia de linfonodo e medula óssea, não foram encontradas formas amastigotas de Leishmania sp. E, por último, o ELISA e a RIFI negativaram para LVC.

Diante dos resultados, para auxiliar no diagnóstico, foi solicitado exame histopatológico da lesão. Para tanto, o paciente foi sedado através da administração de tramadol $3 \mathrm{mg} / \mathrm{kg}$ e acepromazina $0,01 \mathrm{mg} / \mathrm{kg}$, ambos por via intramuscular, e propofol $2 \mathrm{mg} / \mathrm{kg}$ por via intravenosa. Duas amostras foram retiradas da região periférica da lesão em ponte nasal, sendo utilizado um Punch Descartável $6 \mathrm{~mm}$. Após exérese a região foi suturada. A amostra foi armazenada em formaldeído $10 \%$ e encaminhada para análise.

Macroscopicamente, a amostra foi caracterizada como ulcerada, firme e de coloração branca. Microscopicamente, foram observados infiltrado inflamatório predominante em junção dermoepidérmica e apêndices dérmicos, constituído por inúmeros linfócitos e plasmócitos; perda de conformação da junção dermoepidérmica; apoptose de células basais, caracterizadas por aumento de eosinofilia, mas sem formação de corpos coloides; apoptose de queratinócitos; apagamento de membrana basal; melanófagos em derme papilar; incontinência pigmentar e degeneração vacuolar (degeneração hidrópica) de queratinócitos. (Figuras 2A e 2B), sendo essas alterações sugestivas de LED.

Tabela 1. Alterações no perfil hepático e proteinograma de cão, sem raça definida, apresentando lúpus eritematoso discoide.

\begin{tabular}{lll}
\hline Bioquímica sérica & Valor encontrado & Intervalo de referência \\
\hline Fosfatase alcalina & $290 \mathrm{UI} / \mathrm{L}$ & $20-150 \mathrm{UI} / \mathrm{L}$ \\
Proteínas totais & $9,6 \mathrm{~g} / \mathrm{dL}$ & $6,0-8,0 \mathrm{~g} / \mathrm{dL}$ \\
Globulina & $6,5 \mathrm{~g} / \mathrm{dL}$ & $2,7-4,4 \mathrm{~g} / \mathrm{dL}$ \\
Relação A/G* & 0,477 & $0,59-1,1$ \\
\hline
\end{tabular}

*Relação Albumina/Globulina
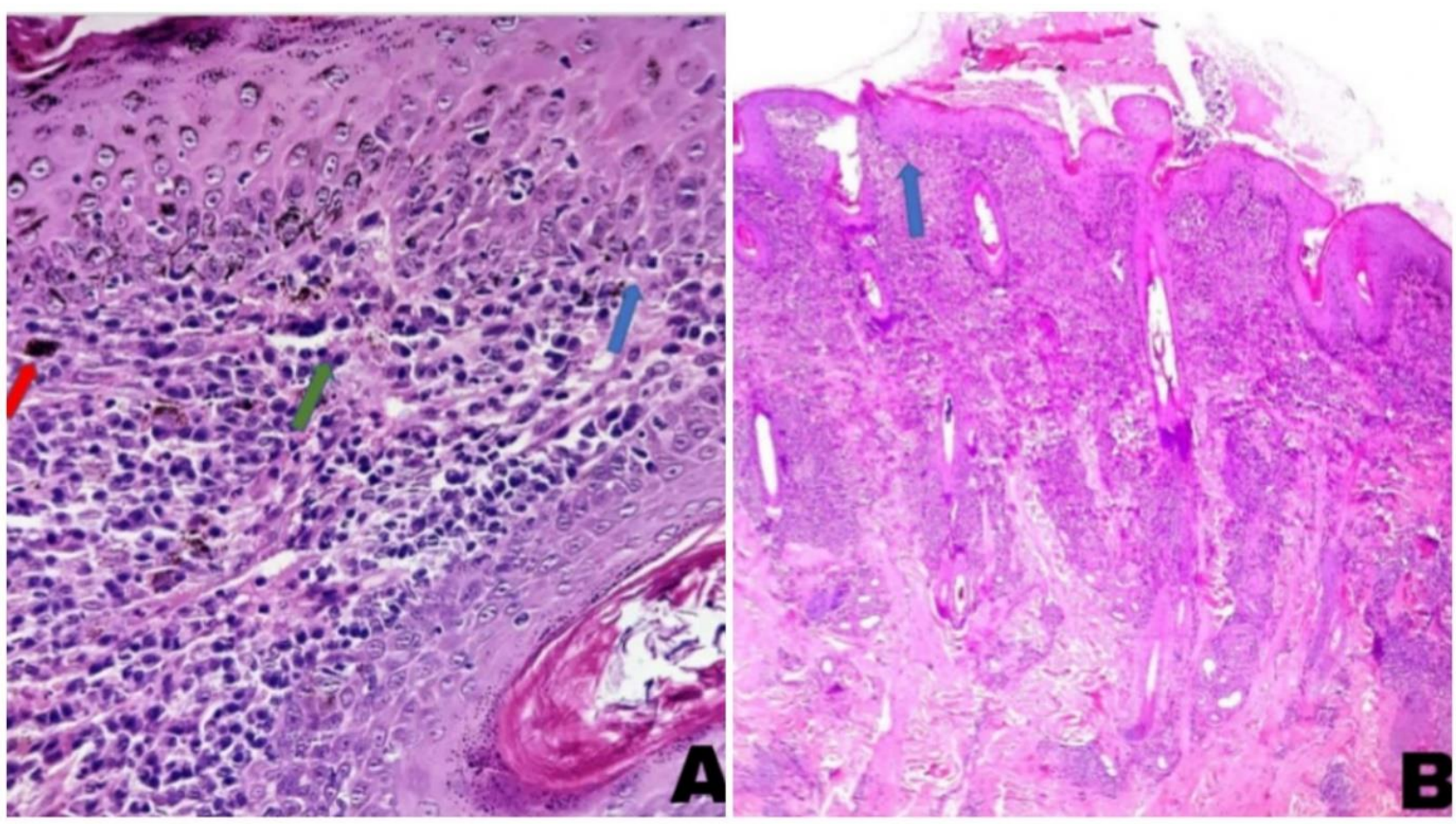

Figura 2. Fotomicrografia de corte histopatológico de pele lesionada de plano nasal de cão apresentando sinais macroscópicos de LED. (A) 40x - Presença de melanófagos em derme papilar (seta vermelha); infiltrado inflamatório formado predominantemente por linfócitos e neutrófilos (seta verde); e apagamento de membrana basal (seta azul). (B) $4 \mathrm{x}$ - Perda de conformação da junção dermoepidérmica (seta azul). Coloração: hematoxilina-eosina. 
Diante do resultado histopatológico, foram iniciadas as terapias sistêmica e tópica do paciente com prednisolona, por via oral, na dose de $1 \mathrm{mg} / \mathrm{kg}$ a cada 24 horas, durante 20 dias; e protetor hepático à base de precursores da glutationa, aminoácidos e vitaminas, por via oral, 2 comprimidos a cada 24 horas, durante 30 dias. Por via tópica, foi prescrita pomada à base de hidrocortisona $1 \%$, vitamina $\mathrm{E} 0,5 \%$ e fator de proteção 45, a cada 12 horas, durante 20 dias. Também foi recomendado que o paciente não fosse exposto ao sol por longos períodos, principalmente nos horários de maior incidência solar.

Foi solicitado à tutora que retornasse após o período de 20 dias para reavaliação do paciente. No retorno, constatou-se melhora considerável da lesão em ponte nasal. A tutora demonstrou satisfação com a evolução do quadro clínico. $O$ tratamento foi bem tolerado pelo paciente, não sendo observadas alterações clínicas decorrentes da terapia.

Foi iniciada a redução gradativa da dose da prednisolona, $0,75 \mathrm{mg} / \mathrm{kg}$, a cada 24 horas, durante 20 dias; depois, $0,5 \mathrm{mg} / \mathrm{kg}$, a cada 24 horas, durante 20 dias, seguido de $0,25 \mathrm{mg} / \mathrm{kg}$, durante 20 dias; por fim, retirou-se completamente o medicamento. O tratamento tópico foi mantido por quatro meses. A tutora foi contactada durante todo o período do tratamento para acompanhamento da evolução do caso.

Após os quatro meses, o animal apresentou remissão total da lesão (Figura 3). Foi recomendada a utilização de fator de proteção 45 sobre a ponte nasal por toda a vida do paciente, bem como cuidados com a exposição solar. Oito meses após o fim do tratamento, a tutora relatou que o animal não apresentou recidiva das lesões durante este período.

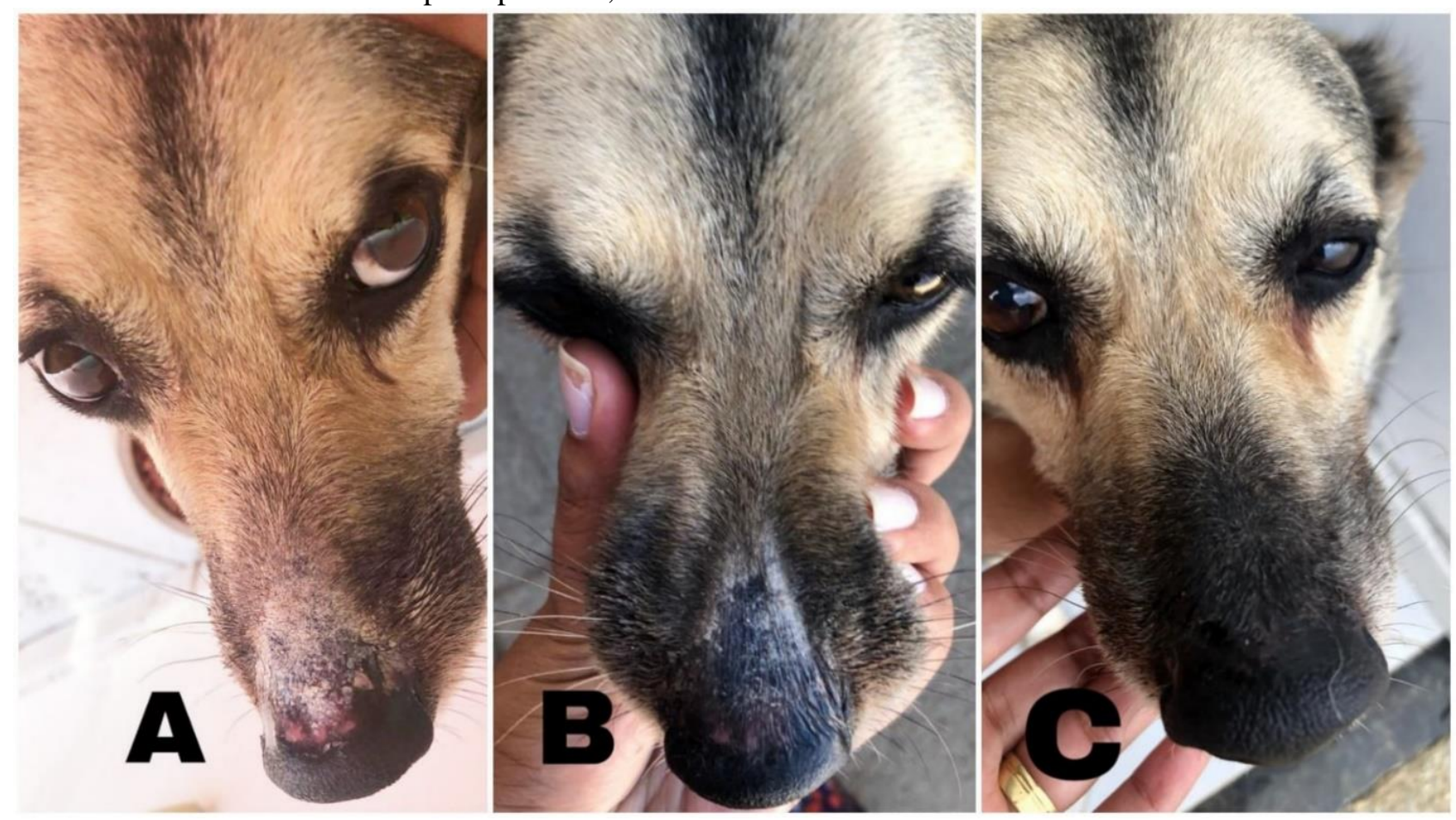

Figura 3. Evolução da lesão decorrente de lúpus eritematoso discoide em cão. A - Paciente após um mês de tratamento. B - Paciente após três meses de tratamento. C - Paciente ao fim do tratamento, com remissão completa da lesão após oito meses.

\section{Discussão}

O LED apresenta, dentre suas características clínicas, manifestações localizadas na região do nariz, podendo ainda, de maneira não muito comum, espalhar-se para a região periocular, membros e mucosas. Essa lesão pode aumentar de tamanho e possuir aspecto ulcerativo e erosivo. No início do quadro clínico, apresenta descamação, discromia e eritema, criando um aspecto de "calçamento de pedras", mas com superfície lisa. Além disso, ocorre uma despigmentação e a lesão adquire uma coloração azul escura ou cinza. Com a progressão da doença, surgem as lesões crostosas e ulceradas (Rossi et al., 2015; Balazs, 2017), o que corrobora os achados encontrados no animal deste relato.

No que diz respeito à epidemiologia, o estudo de Palumbo et al. (2010) relata que a maioria dos casos acontece em cães e fêmeas, sem associação com a raça, pois a maioria dos cães 
não apresentaram raça definida. Com relação à localização das lesões, as áreas mais comuns foram a ponte nasal e região periocular. Todos esses achados condizem com os deste estudo, exceto com relação ao sexo.

Porém, confrontando esses dados, alguns autores fazem uma associação da predisposição racial e o aparecimento da doença (Lima-Verde et al., 2020); além da relação hormonal, com maior caso nas fêmeas (Miller et al., 2013). Neste sentido, o aparecimento do LED no paciente em estudo pode estar relacionado ainda a fatores ambientais, como exposição excessiva ao sol (Tizard, 2009).

No que diz respeito à idade, alguns autores apontam para uma maior frequência nos cães de dois a cinco anos (Larsson e Otsuka, 2000), porém Palumbo et al. (2010) encontraram variações de idade entre 4 e 11 anos. Desse modo, o paciente em questão apresentou a doença já em idade mais avançada, estimada em 12 anos.

Com relação ao diagnóstico, sabe-se a dificuldade de realizá-lo e possível subnotificação do LED (Lima-Verde et al., 2020), tendo em vista a necessidade de anamnese, exame físico, exame de sangue, diagnóstico diferencial, e, para diagnóstico definitivo, o histopatológico, sendo este o teste ouro (Oberkircher et al., 2011).

Sobre isto, na coloração HematoxilinaEosina (HE), as principais alterações são encontradas nas camadas basal e superficial da pele, havendo perda da conformação da junção dermoepidérmica, bem como presença de infiltrado inflamatório de leve a moderado. Entre achados importantes estão a incontinência pigmentar, associada à presença de melanófagos, queratinócitos apoptóticos e degeneração hidrópica (Silva et al., 2018; Ataíde et al., 2019). Neste trabalho, observaram-se melanófagos em derme papilar, infiltrado inflamatório com predominância de linfócitos, plasmócitos e neutrófilos, apoptose de células basais e apagamento da membrana basal com perda da conformação da junção dermoepidérmica, caracterizando a dermatite mista de interface, com degeneração vacuolar; modificações, portanto, indicativas de LED e semelhantes àquelas descritas por Silva et al. (2018) e Ataíde et al. (2019).

Neste caso, ainda foi realizado o diagnóstico diferencial para Leishmaniose Visceral Canina (LVC), que é recomendado pela literatura para áreas endêmicas, visto que LED e
LVC podem cursar com dermatite em ponte nasal e os aspectos histopatológicos nas lesões são semelhantes (De Lucia et al., 2017). Portanto, a biópsia das lesões não é o suficiente para concluir o diagnóstico de LED em áreas endêmicas de LVC, como é o caso de Mossoró-RN, cidade onde o caso relatado ocorreu.

Com relação aos demais exames, embora anemia discreta e trombocitopenia possam estar presentes em casos mais graves, as alterações hematológicas em animais com LED são raras e inespecíficas (Bryden et al., 2005; Olivry et al., 2018), corroborando o encontrado no paciente, que não apresentou alterações hematológicas.

No que tange às bioquímicas séricas, geralmente, não há alterações significativas, exceto a hiperglobulinemia, que pode ser identificada eventualmente (Olivry et al., 2018; Mauldin et al., 2010). O animal apresentou hiperglobulinemia. $\mathrm{O}$ aumento considerável da fosfatase alcalina do paciente, no entanto, não condiz com o observado pelos autores, podendo ser atribuído a uma comorbidade, sendo necessária a investigação.

No que diz respeito à terapêutica, as lesões decorrentes do LED aparentam responder a vários tipos de tratamentos sistêmicos e tópicos. Embora grande parte apresente recidiva após a diminuição das doses (Olivry et al., 2018), e, ainda com a variabilidade, o objetivo é o mesmo: controlar as vias inflamatórias envolvidas na patogênese do LED (Banovic, 2019). Portanto, a escolha do protocolo terapêutico empregado para o paciente levou em consideração o estudo realizado por Jessop et al. (2017), em humanos com LED, e Ettinger e Feldman (2004), em animais, em que foi reportado que o uso de hidrocortisona por via tópica é eficaz para o tratamento da doença.

A utilização de medidas fotoprotetoras é imprescindível para o sucesso terapêutico em casos de LED (Banovic, 2019), visto que a exposição solar é um fator envolvido no desenvolvimento da doença (Ribeiro et al., 2008). Tais medidas envolvem o uso de filtro solar e diminuição da exposição solar, principalmente em horários de maior incidência (Ribeiro et al., 2008; Banovic, 2019). Deste modo, foi formulada uma pomada contendo hidrocortisona, fator de proteção solar e vitamina E; esta última pelo seu efeito antioxidante, que, como relatado por Gorman (1997), tem se mostrado efetiva como adjuvante no controle dos sinais da doença. 
No estudo realizado por Banovic et al. (2016), ciclos curtos de glicocorticoides levaram à melhora do quadro clínico ou remissão completa. A dose preconizada pela literatura é de $2 \mathrm{mg} / \mathrm{kg} / \mathrm{dia}$, entretanto, a maioria dos pacientes apresenta recidivas após a diminuição gradativa das doses (Bryden et al., 2015; Banovic, 2019). No caso do paciente relatado, por apresentar uma alteração prévia da fosfatase alcalina, o tratamento foi iniciado com uma dose de corticoide menor que a recomendada. Além disso, foi prescrito protetor hepático, para auxiliar nesse desajuste. De maneira geral, o tratamento apresentou sucesso, visto que não foi observada recidiva, mesmo com a diminuição progressiva e término do tratamento.

\section{Conclusão}

Há uma necessidade de estabelecer uma rotina e elo entre a clínica e exames laboratoriais, no sentido de diminuir a subnotificação de doenças como o LED, bem como estabelecer tratamentos adequados. De maneira geral, esta discussão vem suscitar a necessidade de atenção por parte dos médicos veterinários quanto à ocorrência de lúpus eritematoso e da importância da realização do exame histopatológico, bem como diagnóstico diferencial para outras doenças que possam cursar com lesões em ponte nasal, como é o caso da leishmaniose visceral canina.

Com relação ao protocolo terapêutico utilizado neste caso, houve eficácia para o tratamento do paciente, que, além de apresentar remissão das lesões, não desenvolveu recidivas, mesmo após a retirada completa do corticoide por vias sistêmica e tópica, apresentando-se, portanto, como uma possibilidade terapêutica.

\section{Conflito de interesse}

Os autores declaram não existir conflito de interesse.

\section{Referências}

Ataíde, W. F.; Silva, V.L.D.; Ferraz, H.T.; Amaral, A.V.C.; Romani, A.F. Lúpus Eritematoso Discoide em cães. Enciclopédia Biosfera, 16(29): 905-1009, 2019.

Balazs, V. Caso clínico: Lupus eritematoso mucocutáneo en un perro. Revista Hospitales Veterinarios - Digital, 9(1): 611, 2017.

Banovic, F.; Linder, K.E.; Uri, M.; Rossi, M.A.; Olivry, T. Clinical and microscopic features of generalized discoid lupus erythematosus in dogs (10 cases). Veterinary Dermatology, 27: 488-e131, 2016.

Banovic, F.; Robson, D.; Linek, M.; Olivry, T. Therapeutic effectiveness of calcineurin inhibitors in canine vesicular cutaneous lupus erythematosus. Veterinary Dermatology, 28(5): 493-115, 2017.

Banovic, F. Canine cutaneous lupus erythematosus: newly discovered variants. Veterinary Clinics of North America: Small Animal Practice., 49(1):37-45, 2019.

Bryden, S.L.; White, S.D.; Dunston, S.D.; Burrows, A.K.; Olivry, T. Clinical, histopathological and immunological characteristics of exfoliative cutaneous lupus erythematosus in 25 German shorthaired pointers. Veterinary Dermatology, 16: 23952, 2005.

De Lucia, M.; Mezzalira, G.; Bardagí, M.; Fondevila, D.M.; Fabbri, E.; Fondati, A. A retrospective study comparing histopathological and immunopathological features of nasal planum dermatitis in 20 dogs with discoid lupus erythematosus or leishmaniosis. Veterinary Dermatology, 28(2): 200-e 46, 2017.

Ettinger, S.J.; Feldman, E.C. Tratado de medicina interna veterinária: doenças do cão e do gato. $5^{\text {a }}$ ed. Rio de Janeiro: Guanabara Koogan, 2004.

Ferreira Filho, S.G.; Fernandes, F.L.; Chamelete, M.O.; Cruzeiro, R.S. Lúpus eritematoso discoide canino: relato de caso. PUBVET, 8(22): 1810, 2014.

Gomes, P.M.S. Lúpus eritematoso cutâneo: manifestações clínicas e análise laboratorial. Revista Sustinere, 3: 3-21, 2015.

Gorman, N.T. Imunologia. In: Ettinger S.J.; Feldman E.C. Tratado de medicina interna veterinária. $4^{\mathrm{a}}$ ed. São Paulo: Manole. 1997. p. 2735-2765p.

Jessop, S.; Whitelaw, D.A.; Grainge, M.J.; Jayasekera, P. Drugs for discoid lupus erythematosus. Cochrane Database of Systematic Reviews, 5(5): CD002954, 2017.

Laflamme D. T. Development and validation of a body condition score system for dogs. Canine Practice., 22(4): 10-15, 1997.

Larsson, C. E.; Otsuka, M. Lúpus Eritematoso Discoide- LED: Revisão e casuística em serviço especializado da Capital de São Paulo. Revisa de Educação Continuada em 
Medicina Veterinária e Zootecnia do CRMV-SP, 3(1): 29-36, 2000.

Lima-Verde, J.F; Ferreira, T.C.; Nunes-Pinheiro, D.C.S. Lupus eritematoso discoide em cão: relato de caso. PUBVET, 14(1): 1-6, 2020.

Mauldin, E.A.; Morris, D.O.; Brown, D.C.; Casal, M.L. Exfoliative cutaneous lúpus erythematosus in German shorthaired pointer dogs: disease development, progression and evaluation of three immunomodulatory drugs (ciclosporin, hydroxychloroquine, and adalimumab) in a controlled environment. Veterinary Dermatology, 21: 373-382, 2010.

Medleau, L.; Hnilica, K. A. Dermatologia de pequenos animais: atlas colorido e guia terapêutico. São Paulo: Roca, 2003. p. 128129.

Miller, W.H.; Griffin, C.E.; Campbell, K.L.; Muller, G.H. Small animal dermatology. $7^{\text {th }}$ ed. St Louis: Elsevier Health Sciences, 2013. 948p.

Oberkirchner, U.; Linder, K. E.; Olivry, T. Successful treatment of a novel generalized variant of canine discoid lúpus erythematosus with oral hydroxychloroquine. Veterinary Dermatology, (23): 65, 2011.

Olivry, T.; Linder, K.E.; Banovic, F. Cutaneous lupus erythematosus in dogs: a comprehensive review. BMC Veterinary Research, 14 (132): 1-18, 2018.

Palumbo, M.I.P.; Machado, L. H. A.; Conti, J.P.; Oliveira, F.C.; Rodrigues, J.C. Incidência das dermatopatias auto-imunes em cães e gatos e estudo retrospectivo de 40 casos de lúpus eritematoso discoide atendidos no serviço de dermatologia da Faculdade de Medicina Veterinária e Zootecnia da UNESP Botucatu. Semina: Ciências Agrárias, 31(3): 739-744, 2010.

Ribeiro, L.H.; Nunes, M.J.; Lomonte, A.B.V.; Latorre, L.C. Atualizações no Tratamento do Lúpus Cutâneo. Revista Brasileira de Reumatologia, 48(5): 283-290, 2008.

Rossi, M.A; Messenger, L.M; Linder, K.E; Olivry, T. Generalized canine discoid lupus erythematosus responsive to tetracycline and niacinamide therapy. Journal of the American Animal Hospital Association, 51(3): 171-175, 2015.

Silva, V.L.; Monteiro, C.L.B.; Silva, M.C.; Carneiro, R.D.; Pereira Júnior, E.R.; Lucena, L.V. Diagnóstico e tratamento de lúpus eritematoso discoide canino: relato de caso. PUBVET, 12(3): 1-6, 2018.

Tizard, I.R. Imunologia veterinária: uma introdução. $9^{\mathrm{a}}$ ed. Rio de Janeiro: Elsevier, 2009. 900p. 